\title{
Impact of Intensified Pharmaceutical Interventions in Medication Adherence in Chronic Kidney Disease Patients
}

\author{
Dilip Chandrasekhar1*, VM Ganesan², Sreelekha Sreekumar', Anuja Pradeep', Asha Susan Geoji', Athira Elezabath George ', \\ Athira $\mathbf{V}^{1}$ \\ 'Department of Pharmacy Practice, Al Shifa College of Pharmacy, Perinthalmanna, Kerala, INDIA. \\ ${ }^{2}$ Consultant Nephrologist, KIMS, Al Shifa Hospital Pvt. Ltd., Perinthalmanna, Kerala, INDIA.
}

\begin{abstract}
Aim: The study aims to evaluate the medication adherence behavior of individuals by using validated questionnaire and to enhance the adherence by using various cost effective interventional methods which likely have a greater effect on the health of patients with chronic diseases than any Improvement in specific medical therapies. Method: The prospective interventional study was carried out for a period one year. The participants selected from the outpatients of nephrology department in a 750-bedded tertiary care hospital in the Malabar region of south India. Patients were provided with a standard validated modified Morisky eight item questionnaire and self-reporting questionnaire assessing medication adherence before and after intervention. The intervention mainly in the form of patient counseling and patient information leaflet was carried out using a proper management plan and with the help of physician and feedback information was collected. Results: Statistically significant association exist between medication adherence before and after intervention $(p<0.001)$. Medication adherence behavior of study subjects has a significant association with age, sex, education and stage of chronic kidney disease. Pharmaceutical interventions impart a significant improvement in the medication adherence
\end{abstract}

behavior. Cost of the treatment, low family income and drug therapy burden have been reported as a main reason for non-adherence. Individual patients have their own preferences for dosage form, frequency of administration, food time etc. Conclusion: The study suggests that the periodic counseling by clinical pharmacist at regular intervals can improve the medication adherence by improving awareness and removing the misconceptions about the disease and therapy.

Key words: CKD, Medication adherence, Drug therapy, Patient counseling and Patient information leaflet, Renal disease.

Correspondence

Dr. Dilip Chandrasekhar, Department of Pharmacy Practice, Alshifa college of Pharmacy, Poonthavanam P.O, Perinthalmanna, Kerala, INDIA.

Phone: +919447252670

Email: dillu7@gmail.com

DOI: 10.5530/jyp.2018.10.46

\section{INTRODUCTION}

Medication adherence can be defined as a condition in which a patient's medication taking behavior coincides with the intention of health advice he or she has been given. Good medication adherence is important to obtain desired therapeutic outcome where as low adherence is considering to be one of the major reasons that not only decreases desired therapeutic outcome but also increases financial burden in individual patients and healthcare system. When it comes to chronic diseases, medication adheres is the key link between treatment and outcome. In case of both developing and developed countries chronic kidney disease, (CKD) which can be defined as an increase in serum creatinine concentrations greater than 1.2 to $1.5 \mathrm{mg} / \mathrm{dl}$, is one of the major public issues. Anemia, secondary hyperparathyroidism, renalosteodystrophy, hypertension and hyperlipidemia, metabolic acidosis, and several other comorbidities involving malnutrition, pruritus and uremic bleeding are can occur as associated with CKD. ${ }^{1}$ These patients are also at increased risk of cardiovascular disease (CVD) such as coronary heart disease (CHD), cerebrovascular disease, peripheral vascular disease, and heart failure. As there is no defined gold standard for measuring adherence so far, it makes difficult not only to carry out the research involving such topics but also comparison of results with other similar researches. Large number of prescribed medicines and frequent dosing changes may contribute to decreased adherence which in turn can lead to drug related morbidities. In case of CKD major goals are to slow down the disease progression and prevent the related complications through careful monitoring. For this adherence to the treatment regimen is necessary. Increased morbidity and mortality in developing countries is mainly due to poor adherence to the therapy regimens. ${ }^{2}$ The study aims to evaluate the medication adherence behavior of individuals by using validated questionnaire and to enhance the adherence by using various cost effective interventional methods which likely have a greater effect on the health of patients with chronic diseases than any improvement in specific medical therapies.

\section{MATERIALS AND METHODS}

This prospective interventional study of one-year duration was conducted in the nephrology department of a tertiary care hospital of Malabar region in South India. A pilot study was carried out to find out the patient's response and attitude towards the study. The study was conducted in 3 phases. In the initial stage, pre-interventional, approximately 950 patients consulted in the department 163 patients were selected based on inclusion-exclusion criteria and informed consent was taken for the study after filtered in pursuance of inclusion exclusion criterions. Nephrology outpatients those underwent the treatment of chronic kidney disease, both male and female patients, who are willing to participate in the study were included in the study while pregnant patients, patients with co morbid conditions like psychiatric illness, cognitive impairment and malignancy, patients of non-Indian origin, patients who provide incomplete information about drug use and patients on the initial stages of CKD (stage 1 and 2) excluded from the study. CKD stage 1 and stage 2 patients excluded because these patients don't have any long term therapy

This is an open access article distributed under the terms of the Creative Commons Attribution-NonCommercial-ShareAlike 4.0 License, which allows others to remix, tweak, and build upon the work non-commercially, as long as the author is credited and the new creations are licensed under the identical terms. 
with medicines. Modified Morisky 8 item Questionnaire (MMQS-8) was used to assess the medication adherence of the patients and a Selfreporting questionnaire in a format of objective type to be filled by patient/bystander was prepared to gather information regarding patient education, living status, family support, availability of medicines, cost of treatment, awareness about disease condition, knowledge about medicines, economic burden medication taking behavior etc. Cranach's a used for internal validation of MMQS- 8 and the self-reporting questionnaire used were validated by experts using test-retest method. During interventional period medication adherence of the patients were classified in to low, medium and high. Based on the overall medication adherence pattern pharmaceutical interventions like patient counseling and patient information leaflets containing general outline information about CKD and importance of medicines were prepared and provide to individual patients. ${ }^{3}$ A feedback form was designed and developed to collect information from subjects regarding their preferences and attitude towards treatment. Telephonic interview was done when necessary. In post intervention phase the individuals were further evaluated for measuring the medication adherence and compared with the previously obtained score. Feedback form was given to the study subjects and information was collected. The collected data were analyzed categorized and entered Ms. Excel format. According to the formulae $\left(n>Z^{2} \times P \times Q\right) / d^{2}$ theminimum sample size for the study was 116 ,

Where $\mathrm{Z}=$ confidence coefficient $(95 \%)$

$\mathrm{P}=$ rate of prevalence $(50 \%)$

$\mathrm{Q}=1-\mathrm{P}$

$\mathrm{d}=$ error of estimates $(9 \%)$

Different tools were used to perform statistical analysis of various data 'Chi square test' for association of qualitative variables under study, 'Independent student $t$ ' test for comparison of demographics details, 'ANOVA' techniques were used for comparison of various parameters, 'Co-relation and regression' analysis was used for quantitative variables and 'Paired $t$ ' test was performed for comparison of variables.

\section{Outline of Study Methodology}

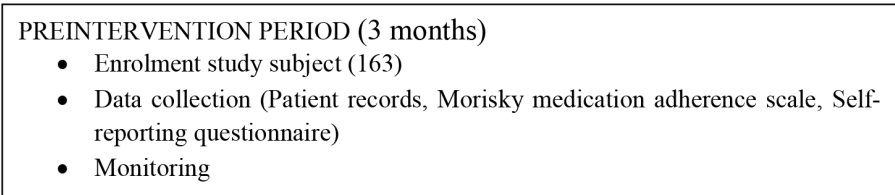

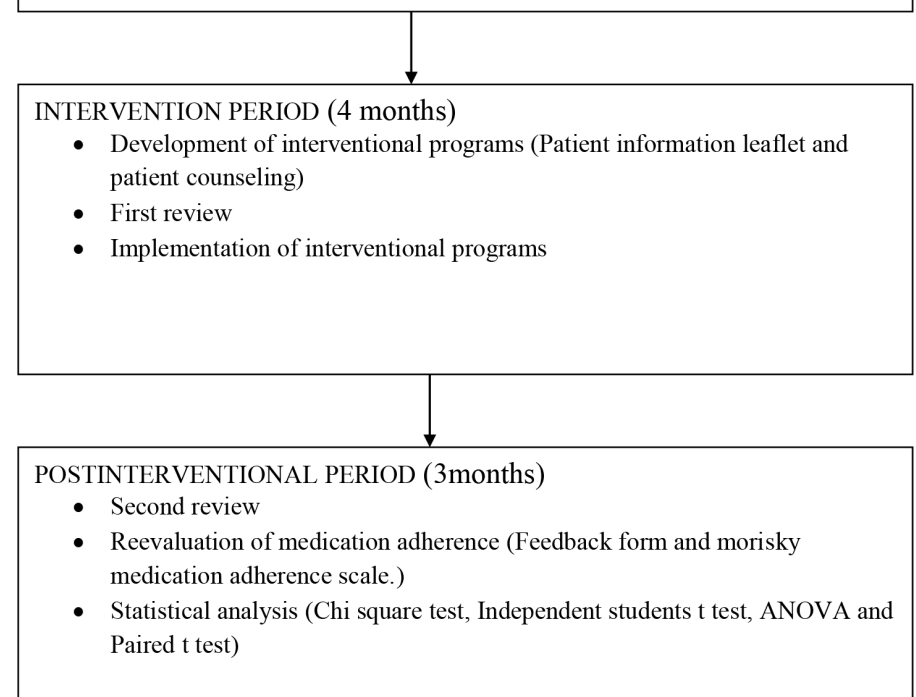

\section{RESULTS}

During the study approximately 950 patients visited Nephrology outpatient department, among them 165 patients selected and enrolled in the study based. Amidst these 163 patients, 14 (8.58\%) were $\geq 76$-yearold, highest number of patients $65(39.87 \%)$ belongs to range $61-75,46$ (28.22\%) patients in range of 46-60. 27 (16.56\%) patients were in group of $31-45,10(6.13 \%)$ in group of $16-30$ and only $1(.61 \%)$ were in range of $0-15$. The correlation between age and adherence, before and after intervention was studied using Karl -Pearson coefficient of correlation and it is find out that there is a significant positive correlation between age and adherence before and after intervention $(p<0.05)$. As age advances, the score obtained by the individuals was also increasing, indicating that medication adherence was decreasing as age increases (Figure $2 \mathrm{a}$ and $2 \mathrm{~b}$ ). In male patients $(\mathrm{n}=91)$ and in female patients $(\mathrm{n}=72)$, there is a statistically significant improvement in high level adherence and low-level adherence after intervention $(p<0.001)$. But comparing to female patients, male patients shows more improvement in medication adherence after the interventional studies (Figure 1). Considering educational status none of the patients were illiterate. $57.05 \%$ people had high school education. $4.97 \%$ were graduates. Only $5.52 \%$ have secondary education. About $13.44 \%$ have lower primary education and $1.22 \%$ has technical education or equivalent. Patients with high school education show higher improvement in medication adherence (Table 2). From total study population $28 \%$ of the patients were insurance holders demonstrated a better adherence than remaining $72 \%$ who were not having any type of insurance policies. Looking forward in to the distribution of study subject based on economic status, $79 \%$ of study subjects belongs to an economic status of average ,17\% individuals belongs to below average economy and only $4 \%$ patients were found to be rich. Comparatively a very few study subjects have a social history of smoking, alcohol or both $12.8 .8 \%, 4.29 \%, 6.134 \%$ respectively. Out of these 163 study subjects, $48 \%$ were in stage 5 of CKD, $32 \%$ in stage 4 of CKD and remaining $20 \%$ were in stage 3 of CKD. None of the study subjects were in stage 1 or 2 of CKD. Majority of the study population, $75.46 \%$, shows medium level of adherence followed by $15.95 \%$, shows low adherence and remaining $8.5 \%$ exhibits high level of adherence. After intervention there is a reduction in medium (56.44\%) and low level $(0.613 \%)$ of adherence and increase in high level adherence (42.94\%) exhibits a statistically significant improvement in medication adherence $(p<0.001)$. Also, comparing to other stages, patients at CKD stage 5 shows more improvement in medication adherence (Table 1). Among total study subjects only $n=111(68 \%)$ patients preferred both

\section{GENDER AND ADHERENCE}

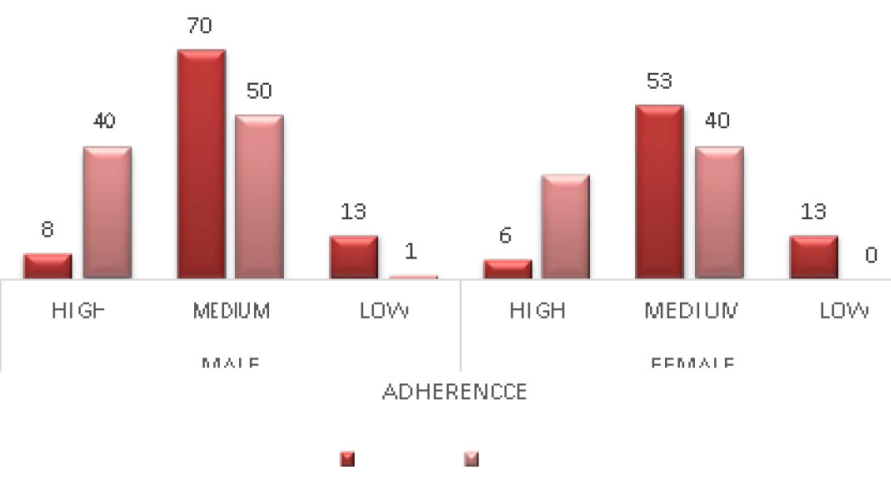

Figure 1: Association of Gender and Adherence.

In male patients $(n=91)$, there is a statistically significant improvement in high level adherence after intervention $(p<0.001)$. 
Table 1: MEDICATION ADHERENCE at VARIOUS STAGES of CKD (before and after intervention).

\begin{tabular}{|c|c|c|c|c|c|c|c|}
\hline \multicolumn{4}{|c|}{ Before intervention } & \multicolumn{4}{|c|}{ After Intervention } \\
\hline MMQS- 1 & CKD - 3 & CKD - 4 & CKD - 5 & MMQS - 2 & CKD - 3 & CKD - 4 & CKD - 5 \\
\hline HIGH & 5 & 1 & 8 & HIGH & 21 & 26 & 23 \\
\hline LOW & 0 & 12 & 4 & LOW & 0 & 0 & 1 \\
\hline TOTAL & 47 & 73 & 43 & TOTAL & 47 & 73 & 43 \\
\hline
\end{tabular}

There is a significant association exist between medication adherence before and after intervention $(p<0.001)$. In medium and low level of adherence there is significant reduction after intervention $(\mathrm{p}<0.001)$. There is significant increase in high level of medication adherence after intervention $(\mathrm{p}<0.001)$

Table 2: Association of Education with Adherence.

\begin{tabular}{cccc}
\hline Education & Adherence & Before & After \\
\hline \multirow{3}{*}{ LP } & HIGH & 2 & 10 \\
& MEDIUM & 16 & 13 \\
& LOW & 5 & 0 \\
UP & HIGH & 1 & 15 \\
& MEDIUM & 26 & 13 \\
& LOW & 1 & 0 \\
HS & HIGH & 5 & 34 \\
& MEDIUM & 72 & 58 \\
& LOW & 16 & 1 \\
HSS & HIGH & 2 & 5 \\
& MEDIUM & 4 & 4 \\
& LOW & 3 & 0 \\
DIPLOMA & HIGH & 1 & 2 \\
& MEDIUM & 1 & 0 \\
& LOW & 0 & 0 \\
DEGREE & HIGH & 3 & 4 \\
& MEDIUM & 4 & 4 \\
\hline
\end{tabular}

A statistically significant improvement can be seen in patients at all level of education $(\mathrm{p}<0.001)$.

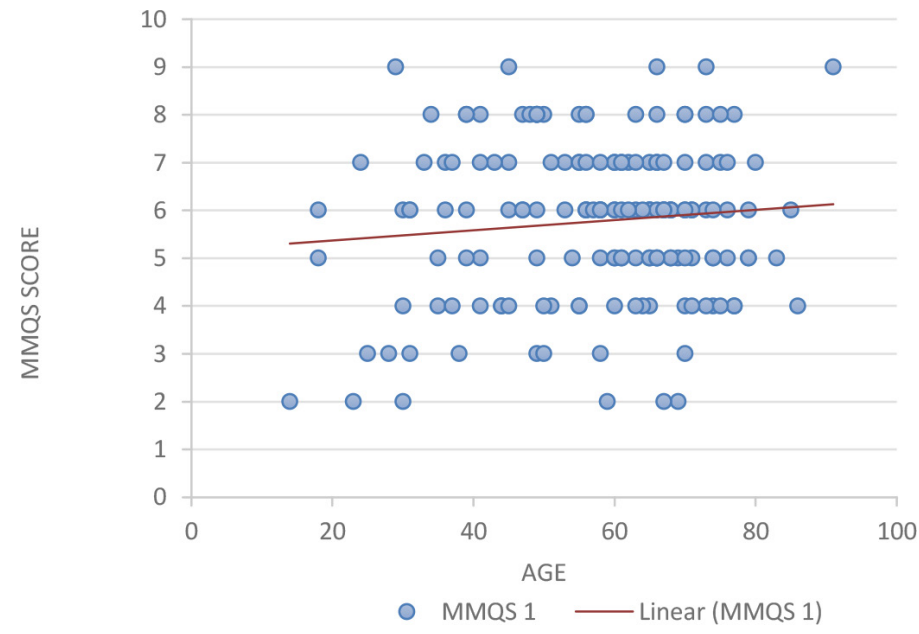

Figure 2a: Association between Age and Adherence before Intervention.

patient counseling and patient information leaflet programs while remaining patients selected either patient counseling $\mathrm{n}=32(20 \%)$ or leaflet $n=20(12 \%)$ as their preference. There is a statistically significant

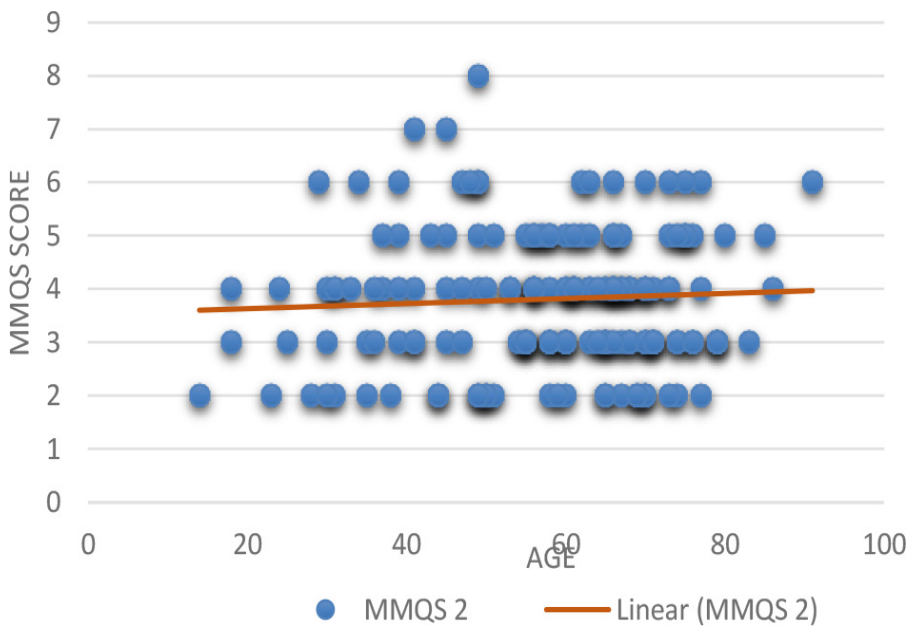

Figure 2b: Association between Age and Adherence after Intervention. There is a significant positive correlation between age and adherence before and after intervention $(p<0.05)$. As age advances, the score obtained by the individuals was also increasing, indicating that medication adherence was decreasing as age increases.

improvement in adherence behavior of patients who supports both type of intervention (Figure 4) but when comparing the intervention preference and change in adherence behavior, medication adherence of the study subjects who preferred patient counseling $(n=32)$ have better improvement (Figure 3). Patients under study reported various factors as reason for no adherence. Among the reasons, majority suggest cost of the drug (77.91\%) as a reason for no adherence was low family income $(77.3 \%)$ was also a reason for poor adherence. It is followed by various other reason for non-adherence such as taste or size of dosage form $(60.12 \%)$, poor awareness about importance of medicines $(44.78 \%)$ and lack of family support (33.35\%). A very few people (16.56\%) suggest side effects of drug as a reason for non-adherence. Majority of the patient population $n=119(73.06 \%)$ preferred to take tablets and capsules. About $n=40(23.53 \%)$ was ready to take all type of dosage form, whereas none of them prefer to take liquid dosage forms. About $60.73 \%$ of patient population suggested once daily dose as a more acceptable frequency for drug administration.

\section{DISCUSSION}

The study was intended to assess and improve medication adherence of the CKD patients in various stages and the study showed that pharmaceutical interventional programs like patient counseling and patient information leaflets alone or together could improve medication adherence in a significant level. A total of 163 were enrolled into the study. The socio demographic details of the study population were slightly different from that of the previous studies. Mean age of the patient in the study 


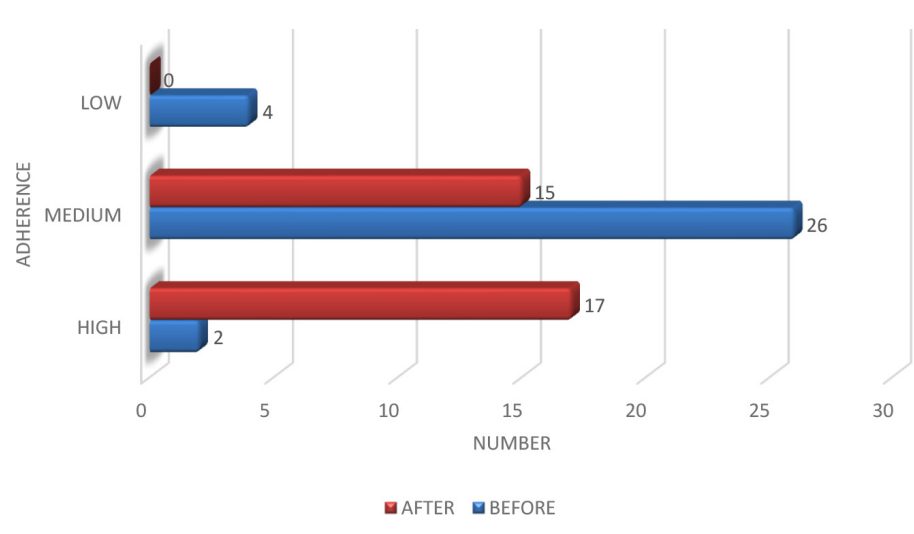

Figure 3: Patient Counseling and Adherence. There is a statistically significant improvement in the adherence behaviour after intervention can be seen $(p<0.001)$

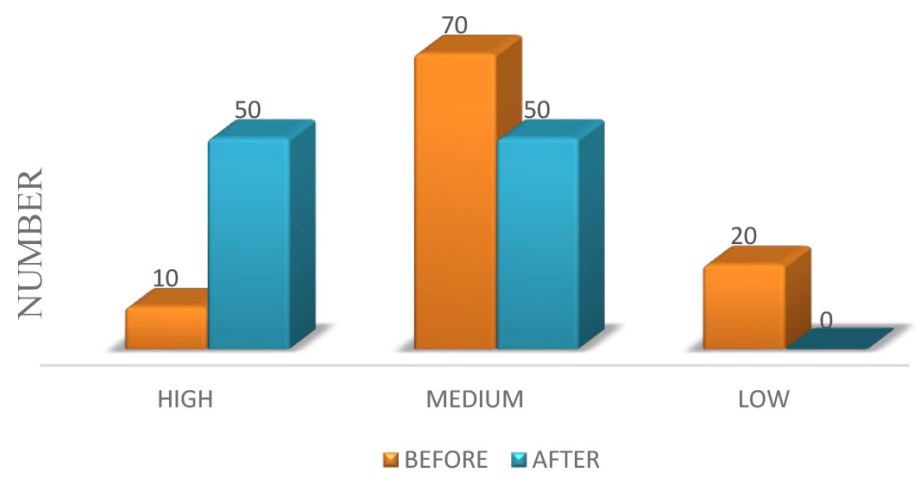

ADHERENCE

Figure 4: Leafelet and Adherence.

There is a statistically significant improvement in the adherence behaviour of the patients who preferred leaflet, after the intervention $(p<0.05)$.

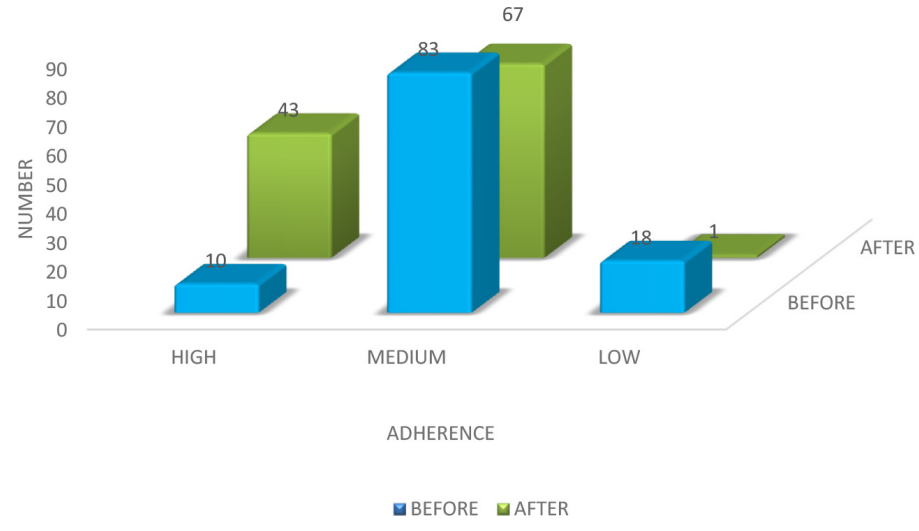

Figure 5: Patient Counseling and Leaflet vs Adherence. After intervention phase there is a statistically significant improvement in medication adherence in patients who preferred both patient counseling and patient information leaflet $(\mathrm{P}<0.001)$

was $57.24 \pm 15.493$, which is slightly greater than that of study conducted ${ }^{1}$ (51 \pm 16.7$)$. There is a prevalence of male patients $(60.4 \%)$, whereas in study conducted ${ }^{1}$ it was $62.7 \%$. Chronic renal failure is categorized in to five different stages based on GFR values. As the disease progress severity of the disease also increases. Patients from stage 1 and 2 were not included in the study as they don't have long term therapy, but the progression of disease mainly prevented by lifestyle modifications. Majority of patients (44.78\%) were on stage 4 , followed by $28.83 \%$ and $26.38 \%$ on stage 3 and stage 5 respectively. Medication adherence behavior of study patients were analyzed using MMQS- 8 item scale which categorizes adherence behavior into three groups low adherence, medium and high adherence. Here, majority of sample $75.46 \%$ have medium adherence $5.95 \%$ have low adherence and $8.5 \%$ have high adherence before the implementation of pharmaceutical interventional programs with a mean MMQS score of $5.77 \pm 1.642$ and these results were contradictory with the results of the study conducted ${ }^{4}$ in which the adherence percentage of study subjects were $51.3 \%, 29.7 \%$ and $19.0 \%$ in high, medium and low level of adherence respectively. This contradiction in the adherence percentage was due to the lack of awareness, poor knowledge about proper usage of medicine, economic problem, treatment cost etc. After implementation of pharmaceutical care programs like patient counseling and patient information leaflet patient in high level of adherence increased to a percentage of 42.94 and patients in low level adherence and medium level adherence decreased to a percentage of 56.44 and 0.613 respectively and the mean MMQS score have been changed to $3.81 \pm 1.279$. This relative change in the adherence behavior indicates an improvement in the medication adherence behavior that occurred as a result of effective pharmaceutical care programs. Extensive studies on adherence behavior of patients at different stages of renal failure were rare and hence a comparison at different aspects was not possible. Within the study patients at stage 5 of CKD exhibits a little more improvement than the other two stages. But results were in contradiction with study conducted. ${ }^{5}$ To a certain extent medication adherence behavior was influenced by gender of patient. The improvement in adherence behavior of male patients was found to be greater than that of females. This difference may be due to difference in educational status, poor family support and awareness, priorities among individuals etc. Level of education have a great impact on adherence behavior, patients with high school education shows good improvement in medication adherence than others which is like study conducted. ${ }^{6}$ A significant positive correlation $(\mathrm{z}=0.376)$ exist between age and adherence. As age increases adherence behavior of study subjects decreases. This was in contradiction with study conducted, ${ }^{7}$ in which they concluded that adherence behavior decreases as age decrease. Among the two intervention programs individual study subjects prefer to choose an intervention or both. About $68 \%$ of patients suggested both patient counseling and information leaflet as an effective method whereas $20 \%$ suggested patient counseling and $12 \%$ suggested leaflet respectively. Within these preference patients supporting patient counseling exhibits more significant improvement in medication adherence which is same as that of the results of study conducted. ${ }^{8}$ In the current study, many patients reported more than two factors as a reason for non-adherence. About $77.9 \%$ of patients' reports cost of treatment as reason for no adherence. Similar observations were also reported in studies conducted. ${ }^{1,9}$ In present study $76.7 \%$ of study population reported burden of medication therapy as a reason like the study conducted. ${ }^{10}$ As Medicines are available in different dosage form they can also influences medication adherence behavior of the patients. About $73.06 \%$ of patients prefer to take tablet and capsules as their option because of ease of consumption also time and frequency of administration have influenced the medication adherence behavior of patients, $60.73 \%$ of patients prefer once daily dosing at food time $(60.75 \%)$ which is Similar to study conducted. ${ }^{11}$

\section{CONCLUSION}

This study explores the impact of a pharmaceutical care based programme for improving the medication adherence in patients with chronic renal failure. Impact of patient counseling was higher in improving the medication adherence. Education and age have great influence on medication 
adherence. Ranging from CKD stage 3 to 5, the adherence behaviors of study subjects were different. As stage of CKD progress, the adherence behaviors of study population were increasing. Various factors were reported as a reason for non-adherence. The treatment cost and low family income were the major reason for non-adherence. Along with these, availability of drug, side effect, taste and size of dosage form, burden of therapy etc. were also reported as reason for non-adherence.

\section{ACKNOWLEDGEMENT}

We are most grateful to all participants of this project and their next of kin.

\section{CONFLICT OF INTEREST}

The authors declare no conflict of interest.

\section{ABBREVIATIONS}

CHD: Coronary Heart Disease; CKD: Chronic Kidney Disease; CVD: Cardio Vascular Disease; MMQS-8: Modified Morisky 8 item Questionnaire; GFR: Glomerular Filtration Rate.

\section{REFERENCES}

1. Magacho EJC, Riberio LC, Chaoubahz A, Bastos MG. Adherence to Drug Therapy in Kidney Disease. Braz J Med Biol Res. 2011;43(3):258-62.

2. Shi L, Liu J, Fonseca V, Walker P, Kalsekar A, Pawasakar M. Correlation between Adherence Rates Measured by MEMS and Self-Reported Questionnaires: A Meta-Analysis. HQLO. 2010;8(1):99-3.

3. Fulmer TT, Feldman PH, Kim TS, Carty B, Beers M, Molina M, et al. An intervention study to enhance medication compliance in community dwelling elderly individual. JAMA. 2007;10(5):3273-7.

4. Fairman KA, Motheral B. Evaluating medication adherence: which is right in your programme? J Manag Care Spec Pharm. 2000;6(6):499-506.

5. Murray MD, Young J, Hoke S, Tu W, Weiner M, Morrow D, et al. Pharmacist intervention to improve medication adherence in heart failure: $A$ randomized control trial. Ann Intern Med J. 2007;146(10):714-25.

6. Hincapie A, Boesen KP, Warholak T, Taylor AM. Understanding reason for nonadherence to medication in a medicare part $D$ beneficiary sample. J Manag Care Spec Pharm. 2015;21:391-5.

7. Rolinick S, Pawloski P, Hedblom BD, Bruzek R. Patient characteristics associated with medication adherence. Clin Med Res. 2013:11(2):54-65.

8. Bouv ML, Heerdink ER, Urquhart J, Grobbee DE, Hoe AW, Leuken HGM. Effect of pharmacist led intervention on diuretic compliance in heart failure patients: a randomized control study. J Card Fail. 2003;9(5):404-11.

9. Tazi MA, Khalils A, Lahmouz F, Arrach ML, Chaouki N. Risk factors for hypertension among the adult Moroccan population. East Med Health J. 2009;15(4):827-41.

10. Neri L, Martini A, Miglior Dialisi study group. Regimen complexity and prescription adherence in dialysis patient. Am J Nephrol. 2011:34(1):71-6.

11. AL-Hajje A, Awada S, Rachidi S, Zein S, Bawab W, Salameh P. Factors affecting medication adherence in Lebanese patient with chronic disease. Clin Med Res. 2013;11(2):54-65.

Article History: Submission Date : 27-10-2017 ; Revised Date : 27-01-2018; Acceptance Date : 23-02-2018.

Cite this article: Chandrasekhar D, Ganesan VM, Sreekumar S, Pradeep A, Geoji AS, George AE, Vipinachadran A. Impact of Intensified Pharmaceutical Interventions in Medication Adherence in Chronic Kidney Disease Patients. J Young Pharm. 2018;10(2):208-12. 